\title{
Archéopages
}

Archéopages

Archéologie et société

$36 \mid 01 / 2013$

Exotismes

\section{Boissons et aliments exotiques en Gaule romaine. L'apport du mobilier amphorique}

Séverine Lemaître

\section{(2) OpenEdition}

1 Journals

Édition électronique

URL : https://journals.openedition.org/archeopages/211

DOI : 10.4000/archeopages.211

ISSN : 2269-9872

Éditeur

INRAP - Institut national de recherches archéologiques préventives

Édition imprimée

Date de publication : 1 novembre 2013

Pagination : 28-35

ISSN : 1622-8545

\section{Référence électronique}

Séverine Lemaître, « Boissons et aliments exotiques en Gaule romaine. L'apport du mobilier

amphorique », Archéopages [En ligne], 36 | 01/2013, mis en ligne le 01 janvier 2015, consulté le 21

janvier 2022. URL : http://journals.openedition.org/archeopages/211 ; DOI : https://doi.org/10.4000/ archeopages. 211 
Des études sociologiques relativement récentes ont abordé l'exotisme associé aux pratiques alimentaires : c'est en particulier le cas de celles de Faustine Régnier. Cette sociologue a travaillé sur la question de l'exotisme culinaire en France et en Allemagne entre 1930 et aujourd'hui à partir de près d'un millier de recettes de cuisine publiées dans quatre journaux de la presse féminine (Régnier, 2004). Après une réflexion à propos de la notion d'exotisme, qu'elle qualifie de «problématique» et «floue », elle indique que le terme a entretenu dès le départ un lien privilégié avec différentes denrées, y compris alimentaires. « Ainsi, à l'origine, le mot s'applique aux productions d'un sol étranger, le plus souvent des plantes, que l'on retire de leur monde d'origine pour les apporter dans le monde de celui qui parle ou écrit. » (Régnier, 2006, p. 2).

Si la démarche s'applique bien évidemment à un autre contexte historique, économique et social, à savoir la France et l'Allemagne à l'époque contemporaine, la lecture des travaux de Faustine Régnier fait surgir des parallèles intéressants avec la période antique.

En effet, par les denrées qu'elles transportaient dans l'Antiquité, les amphores témoignent, selon les lieux et les époques, de pratiques alimentaires spécifiques là où elles sont mises au jour. Dans le centre et le nord de la Gaule, la découverte de ces conteneurs atteste bien sûr l'importance et la généralisation de la consommation du vin au sein des populations aisées durant les deux derniers siècles avant notre ère, mais également l'introduction progressive de nouveaux ingrédients dans la cuisine, comme l'huile d'olive, les sauces et conserves de poisson et les fruits, après la conquête romaine.

Une vaste enquête menée sur les assemblages d'amphores, d'abord sur l'axe Rhône-Rhin, puis étendue à d'autres régions comme le centre-ouest et le nord-est de la Gaule, a montré la présence d'arrivages réguliers de récipients fabriqués dans différentes régions du bassin oriental de la Méditerranée entre le début de l'époque augustéenne et la fin du $\mathrm{III}^{\mathrm{e}}$ siècle (Lemaître, 2002). Leur origine lointaine et la spécificité des denrées qu'elles ont transportées conduisent de manière légitime à les considérer comme des produits que l'on pourrait qualifier d'exotiques.

En effet, l'adjectif « exotique » est lié à une notion relative qui convoque l'idée de distance et celle d'altérité (Régnier, 2004). Un objet, une denrée, une contrée sont exotiques parce qu'ils sont éloignés géographiquement du point de vue de celui qui établit le constat.

\section{Exoticus : recherche chez les auteurs latins}

Le terme « exotique » vient du latin exoticus

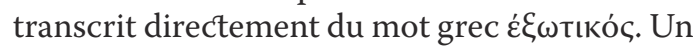
dépouillement systématique réalisé sur l'ensemble des textes de 35 auteurs de langue latine montre clairement que ce mot est rarement employé1. Seules cinq occurrences ont pu être trouvées. La plus ancienne se rencontre dans la pièce Les
Ménechmes de Plaute et correspond à une mention géographique désignant la Grande Grèce : « [...] Graeciam [...] exoticam [...] » (Plaute, Men., 2, 1, trad. A. Ernout), mais peut-être aussi la Grèce d'Asie plus lointaine?

La deuxième citation apparaît dans le livre XIII de l'Histoire naturelle de Pline l'Ancien. Elle est liée à l'interdiction à Rome, au début du ${ }_{\mathrm{II}}{ }^{\mathrm{e}}$ siècle avant notre ère, de l'usage de parfums ou d'onguents qualifiés d'exotiques : «[...] censores edixisse ne quis venderet unguenta exotica; sic enim appellavere. » (Pline, HN, XIII, 5). L'auteur signale en outre quà son époque, certaines personnes ajoutent ces préparations aux boissons, pour leur conférer parfum et amertume.

Une troisième mention concerne à nouveau le domaine de la toilette. Il s'agit d'un passage de l'Apologie d'Apulée où il est question de soin et de nettoyage des dents à l'aide d'une poudre exotique : «[...] exotico pulvere dentis emaculet $[. .]$. (Apulée, Apol., 8, 2). Toujours chez Apulée, mais cette fois dans le texte des Métamorphoses, on trouve à deux reprises le terme exotique. Le premier cas est proche du mot grec exoticos et se comprend au sens «d'étranger »: «[...] siquid exotici ac forensis sermonis rudis locutor offendero » (Apulée, M., 1,1,5). Le personnage d'Apulée s'excuse à l'avance des impairs qu'il pourrait commettre quant aux us et coutumes d'un pays qui n'est pas le sien. Le mot exotique apparaît à nouveau, toujours dans le texte des Métamorphoses, mais cette fois à propos de pratiques culinaires. Il qualifie une sauce accompagnant un poisson que l'on propose, parmi d'autres mets, à Lucius changé en âne: « [...] carnes lasere infectas, altitia inspersa, pisces exotico iure perfusos » (Apulée, M., 10, 16, 5). L'adjectif prend ici aussi le sens d'étranger, d'inhabituel.

Enfin, la dernière occurrence trouvée appartient au texte des Nuits attiques d'Aulu Gelle. Dans le livre XIII, l'auteur évoque la manière dont Aristote a désigné son successeur parmi deux candidats, Théophraste, né à Lesbos et Ménédème, originaire de Rhodes. Aristote dit que le vin qu'il boit ne lui convient pas et qu'il préférerait quelque vin étranger de Rhodes ou de Lesbos : « [...] exoticum vel Rhodium aliquod vel Lesbium » (Aulu Gelle, Nuits attiques, XIII, 5). On lui apporte les deux vins à goûter, après quoi celui-ci indique sa préférence pour le vin de Lesbos, à cause de sa douceur comparé à celui de Rhodes, qualifié de fort et agréable : «[...] firmum [...] et iucundum [...]», dévoilant habilement sa préférence pour Théophraste. Le texte d'Aulu Gelle mentionne ainsi de manière explicite le caractère exotique des vins de Rhodes et de Lesbos, y compris aux yeux du grec Aristote.

L'étude des rares mentions du mot « exotique » chez les auteurs latins montre que le terme, toujours sous la forme d'un adjectif, est appliqué à des parfums, des contrées, des préparations liées à la toilette et à certains vins aux qualités 


$$
\begin{array}{ll}
y & 0 \\
d & 0 \\
y & y \\
y-1 & y
\end{array}
$$


particulières. Du point de vue du sens, il est synonyme d'étranger et de différent, peut-être même de mystérieux dans le cas de la poudre destinée aux soins dentaires. Le faible nombre d'occurrences du mot dans les sources latines ne signifie pas que l'idée qu'il traduit soit absente des propos des Anciens, mais plutôt que d'autres mots lui ont été préférés. En effet, il semble par exemple que l'adjectif peregrinus signifiant étranger ait été plus régulièrement utilisé par les auteurs de langue latine. Il est d'ailleurs souvent traduit par exotique !

À plusieurs égards, les amphores de

Méditerranée orientale et les produits qu'elles ont apportés en Italie et en Gaule durant le HautEmpire, témoignent de l'intégration de denrées exotiques au sein des pratiques alimentaires d'une partie de la population. C'est ce que nous allons essayer de montrer en prenant quelques exemples issus de découvertes archéologiques anciennes ou plus récentes en lien avec deux catégories de denrées, tout d'abord le vin, et ensuite les dattes. Les recherches ont été réalisées à partir des amphores elles-mêmes, en combinant les inscriptions peintes qu'elles ont pu conserver et les restes carpologiques. Les conclusions que nous pouvons établir reposent sur l'idée qu'un type d'amphore était destiné au transport d'un produit particulier. Si ce principe peut être partiellement remis en question à certaines périodes ou dans certaines régions, il reste, nous semble-t-il, largement valable dans le cas des productions amphoriques du Haut-Empire ${ }^{2}$.

\section{Des vins étrangers importés en Italie et en Gaule}

Le livre XIV de l'Histoire naturelle de Pline nous livre un classement précis des différentes espèces de vignes et de vins connues dans le monde antique $\mathrm{au}_{\mathrm{I}}{ }^{\mathrm{er}}$ siècle. Après une présentation hiérarchisée des vins italiens, l'auteur réserve un paragraphe spécial aux vins d'outre-mer transmarina en commençant par les plus célèbres (Pline, $H N$, XIV, 73 à 76). Il cite une trentaine de crus naturels que leur désignation permet de localiser tout autour du bassin oriental de la Méditerranée, depuis l'Égypte jusqu'à la Propontide (actuelle mer de Marmara) et l'Eubée, en passant par le Levant.

Cette profusion n'est pas étonnante dans la mesure où le bassin oriental de la Méditerranée offre des conditions naturelles particulièrement favorables à la culture de la vigne, implantée un peu partout, depuis les grandes vallées de l'Ouest anatolien et les îles, jusqu'aux étroites plaines littorales de la côte levantine et aux territoires plus vastes de l'Égypte romaine.

De la liste énoncée par Pline, seule la consommation d'un petit nombre de ces vins est attestée en Gaule. L'identification de ces derniers s'est faite grâce à des recoupements entre les sources documentaires, en particulier la morphologie des amphores et leur fabric, les dipinti, ainsi que les sources anciennes.

La présence de vins étrangers à la table des habitants de Gaule romaine est un processus engagé de manière très progressive dès les périodes classique, puis hellénistique dans les sites du sud de la Gaule, plus exceptionnellement dans le Nord. Cependant, il faut attendre le $\mathrm{II}^{\mathrm{e}}$ siècle avant notre ère pour voir des importations plus régulières de vins grecs en Gaule septentrionale, et surtout la période impériale pour remarquer un accroissement des arrivages, notamment à partir de l'époque augustéenne (Lemaître, 2005).

Toutes les régions indiquées en brun sur la carte [ill. 1] ont fourni des vins de qualité très différente, dont l'échelle des prix à la vente devait rendre compte, même si les indices concernant cet aspect sont minces (Lemaître, à paraître).

Seul le vin de Chios, dont les amphores ont été identifiées dans quelques ensembles de Gaule romaine et plus récemment à Poitiers/Lemonum (Lemaître, 2012, p. 188-189) est qualifié par les auteurs anciens de grand cru, qui peut même rivaliser avec le Falerne (Pline, $H N$, XIV, 95-96 ; Tchernia, 1986, p. 33 et surtout p. 100-101).

Les autres vins du bassin oriental de la Méditerranée importés en Gaule et originaires de Cos, de Rhodes, de Crète ou de Cnide sont des vins doux et/ou liquoreux de qualité moyenne. Certains appartiennent à la catégorie du passum et d'autres à celle des vina salsa ou tethalassomenoi, dénommés ainsi, car on y ajoutait de l'eau de mer afin de les stabiliser ${ }^{3}$. Cela a sans doute joué un rôle déterminant dans leur commercialisation vers la partie occidentale de la Méditerranée. Le SudOuest anatolien a fourni les plus grandes quantités d'amphores vinaires grecques importées en Gaule entre 30 avant notre ère et la fin du $\mathrm{III}^{\mathrm{e}}$ siècle.

Nous proposons de présenter successivement trois exemples de ces vins, dont le caractère exotique ne fait plus de doute, commercialisés dans des amphores mises au jour dans trois sites différents : du vin de Cos à Troyes, du passum crétois à Orange et du vin doux de Cnide à Toulon.

\section{Du vin de Cos dans la capitale des Tricasses}

Les amphores de type Dressel 2/4 à anse bifide, de Cos et de la région proche, constituent un des groupes de production le mieux représenté dans les importations de Méditerranée orientale en Gaule, sans doute dès le milieu du i ${ }^{\text {er }}$ siècle avant notre ère et jusqu'au début du II $^{\mathrm{e}}$ siècle. En témoignent les données collectées à Augst (Martin-Kilcher, 1994, p. 344-345) ou dans la vallée du Rhône (Lemaître, 2002, p. 220).

Une opération archéologique menée de 2004 à 2006 par l'Inrap, sous la direction de Philippe Kuchler au cœur de la ville antique de Troyes, place de la Libération, a révélé les vestiges d'une insula à vocation artisanale, puis domestique s'échelonnant sur plus de deux siècles (Delor Ahü, Roms, 2007). La fouille a permis la collecte d'un mobilier céramique abondant au sein duquel les amphores importées de la vallée du Rhône et de l'espace méditerranéen jouent un rôle modeste avec 43 individus $^{4}$ sur un total de près de 2500. 
Pourtant, les conditions environnementales spécifiques du site, en l'occurrence le substrat argileux associé à une humidité importante, font de ces amphores un ensemble intéressant. Elles ont en effet permis la conservation d'inscriptions peintes sur le col de plusieurs conteneurs.

Ainsi, un col d'amphore de type Dressel 2/4 avec un dipinto [ill. 2] a-t-il été mis au jour dans le comblement d'une fosse dépotoir (3097Fo), localisée dans l'angle sud de la cour associée à une grande maison rectangulaire installée dans un îlot urbain. Il est possible que cette fosse soit aménagée entre la phase 3 et la phase 4 du quartier antique, ou au tout début de la phase 4 avant que la cour ne soit définitivement close ${ }^{5}$. Les couches archéologiques de la phase 4, scellées par un incendie, rassemblent une partie des mobiliers consommés dans le quartier entre 60-65 et circa 90 (Delor-Ahü, Roms, 2007, p. 71). Le comblement de la fosse a livré un peu de faune et 458 restes de céramiques, dont 88 fragments d'amphores, soit sept individus en NMI, établi à partir des bords, sans pondération. L'amphore de Cos ou de sa région proche était associée à des tessons d'amphores de Bétique, vallée du Guadalquivir, destinées au transport d'huile et de defrutum, une amphore de Gaule narbonnaise et au moins deux amphores d'origine lyonnaise.

L'amphore de type Dressel 2/4 présente un col légèrement conique sur lequel viennent se fixer des anses de section bifide remontantes et dont la partie sommitale est pincée. La morphologie du col doit être rapprochée des productions datées vers le milieu du $\mathrm{I}^{\mathrm{er}}$ siècle. Comme souvent sur les amphores orientales de ce type, la base du col est nettement marquée par un sillon horizontal. Des restes de poix étaient visibles sur la paroi interne de l'amorce de la panse. Son origine orientale est établie à partir d'observations comparatives, à la loupe, de la pâte de l'exemplaire mis au jour à Troyes et de celle de plusieurs récipients découverts à Lyon, et dont l'origine orientale a été confirmée par analyse chimique (Becker et al., 1986)'

Une inscription, faite à la peinture noire en caractères latins sur au moins deux lignes, est conservée dans la partie centrale de l'épaulement. Sur la première ligne, on peut lire : COUM qui désignerait ici le contenu de l'amphore : le cru de Cos. L'appellation est connue par plusieurs dipinti sur des amphores Dressel 2/4 découvertes à Pompéi et Herculanum 7 . L'origine orientale de ces amphores avec inscription COUM aurait besoin d'être confirmée, premièrement parce que les potiers italiens ont imité le modèle Dressel 2/4 originaire d'Orient, de manière très fidèle, deuxièmement, car certains auteurs comme Caton ont livré des recettes pour imiter le vin de Cos avec du raisin italien (Caton, De l'agriculture, 112 ; Pline, $H N$, XIV, 79). Enfin, nous avons déjà évoqué les phénomènes de réutilisation.

Les traces d'une lettre, à peine visible en dessous $\mathrm{du} \mathrm{C}$, indiquent peut-être l'existence d'une seconde ligne d'inscription, mais dont nous ne pouvons rien dire.

D'après les sources textuelles anciennes, le cru de Cos appartient à la catégorie des tethalassomenoi, autrement dit des vins préparés à l'eau de mer (Pline, NH, XIV, 78). Chez Dioscoride, le vin de Cos est associé à celui de Clazomènes : «Le Cos et le Clazomènes, coupés d'eau de mer, se gâtent facilement, produisent des ballonnements, troublent le ventre et sont mauvais pour les nerfs.» (Dioscoride, v. 6, 9-10 et André, 1958, p. 112-113). Il s'agit de vins particuliers préconisés par les médecins antiques dans le traitement de certaines affections (Tchernia, 1986, p. 105-106).

Le site de Troyes, place de la Libération, a également livré d'autres conteneurs d'origine orientale, en particulier des amphores de type rhodien à anse en forme de corne largement importées dans l'ensemble des Gaules et Germanies durant le Haut-Empire (Lemaître, 2002, p. 221, 223224 ; Martin-Kilcher, 1994, p. 348-350).

Des vins au goût spécifique : l'exemple du passum

Une inscription peinte sur le col d'une amphore de type rhodien appartenant au mobilier de la maison de Ménandre à Pompéi met en relation cette catégorie de conteneur avec une boisson spécifique, le passum (A. Maiuri, La casa del Menandro, 1933, cité par Martin-Kilcher, 1994, p. 349, fig. 133). Le dipinto se développe sur deux lignes et mentionne de manière explicite sur la première : «PASS(um). RHOD(ium) », passum des Rhodiens.

Pline parle de ce vin dans un paragraphe consacré aux vins de liqueur, aux côtés des vins cuits. Le passum est obtenu à partir de raisins séchés au soleil (Pline, HN, XIV, 8o). Comme le vin de Cos, celui des Rhodiens est préparé à l'eau de mer, un mode de préparation conférant à cette boisson un goût sans doute particulier.

Ce type d'amphore était produit dans l'île de Rhodes et dans les territoires de la Pérée rattachés administrativement à Rhodes. Le passum rhodien a remporté un vif succès chez les consommateurs gallo-romains à l'époque impériale, comme le prouvent les très nombreuses découvertes d'amphores de ce type sur tout le territoire gaulois.

À partir des années 30-50, un vin venu de Crète fait son apparition en Gaule. Il s'agit également d'un vin doux, appelé passum chez les auteurs latins (Pline, $H N, \mathrm{XIV}, 81$; nombreuses autres références dans Marangou-Lerat, 1995, p. 15-17). Chez Galien, médecin originaire de Pergame, exerçant à Rome au ${ }_{\text {II }}{ }^{\mathrm{e}}$ siècle, le vin doux de Crète entre dans la composition de nombreux médicaments (Marangou-Lerat, 1995, p. 19).

En Gaule, le passum crétois arrive majoritairement par le biais de conteneurs dont la morphologie des anses, dotées de cornes, rappelle celle des amphores de type rhodien. Il s'agit des amphores Dressel 43 ou AC4 (Marangou-Lerat, 1995, p. 84 et suivantes). Les types $\mathrm{AC}_{1}, \mathrm{AC}_{2}$ et $\mathrm{AC}_{3}$ sont également attestés, mais de façon plus discrète, dans les sites de l'axe rhodanien ainsi qu'à Narbonne ou encore Angers 
(Lemaître, à paraître).

À titre d'exemple, un col d'amphore crétoise $\mathrm{AC}_{4}$, découvert à Orange, présentait sur l'épaulement un graffite réalisé à la pointe sèche en caractères latins, peut-être par son propriétaire. Il mentionne explicitement le contenu de l'amphore [ill. 3]. En effet, on peut lire aisément sur deux lignes : PASSUM VIN(um).

Le col conique ainsi que la forme des anses nous conduisent à rapprocher l'amphore mise au jour à Orange des échantillons provenant de l'atelier de production d'Héracléion, localisé sur la côte nord de la Crète (Marangou-Lerat, 1995, pl. XIX, fig. 70, A 127-A 129).

\section{Des amphores cnidiennes à Telo Martius}

Le vin de Cnide arrive aussi en Gaule par le biais des amphores tardo-cnidiennes. Une importante série d'amphores de Cnide de plus d'une centaine d'individus a été mise au jour à Toulon/Telo Martius à l'occasion des fouilles de la zone Lafayette (Imbert, 2002, p. 72). Les conteneurs proviennent d'une cave incendiée, interprétée comme la resserre ou la réserve d'un cabaretier, dont la destruction a été datée de la fin du $\mathrm{II}^{\mathrm{e}}$ siècle. L'ensemble a fait l'objet d'une étude minutieuse de la part de Ernest Louis Imbert publiée en 2002. Ce lot est d'autant plus exceptionnel que le vin de Cnide n'apparaît en Gaule que de manière anecdotique à partir de l'époque augustéenne (Lemaître, 2002, p. 221).

La planche présentée reprend quelques-uns des dessins publiés, dont une proposition de reconstitution graphique de la forme complète, associant différentes parties de plusieurs amphores, aucun récipient n'ayant pu être remonté [ill. 4]. Toutefois, le dessin est cohérent dans la mesure où cette forme, marquée par un élargissement de la panse dans sa partie inférieure, correspond à un exemplaire intact découvert à Saint-Romainen-Gal dans le comblement d'une cave daté de la fin du $\mathrm{II}^{\mathrm{e}}$ ou du début du $\mathrm{III}^{\mathrm{e}}$ siècle (Leblanc, Desbat, 1992).

Une trentaine de ces récipients trouvés à Toulon portait, à la base du col et sur l'épaulement, une inscription peinte en rouge sur deux lignes, dont seule la première nous est lisible. Il s'agit des trois lettres grecques : $\Gamma \Lambda \Upsilon$, la forme abrégée de l'adjectif $\gamma \lambda u \kappa u ́ c$, signifiant doux, sucré. Cet adjectif s'applique aussi au passum de Crète (CIL, IV, 6324 ; Tchernia, 1986, p. 244 pour les références précises).

Pline mentionne également le protopos de Cnide, un genre de passum, mais de mère goutte ${ }^{8}$ (Pline, HN, XIV, 75 et 85 et commentaires p. 117). Les sources anciennes associent volontiers le vin de Cnide à l'usage médical.

La découverte à l'époque impériale de nombreuses amphores à vin originaires du monde grec montre l'intérêt que suscitaient, chez les habitants de la Gaule romaine, ces vins sucrés aux préparations spécifiques. Leurs qualités gustatives, associées à une origine lointaine, en font des vins particuliers que seules les élites pouvaient sans doute s'offrir, même si certains de ces vins comme le passum crétois semblaient, en Italie du moins, plus abordables (Tchernia, 2011). Il ressort également que le degré d'exotisme des produits est relativement indépendant de la valeur intrinsèque des denrées importées, pourvu qu'elles viennent de loin.

\section{Des importations de fruits exotiques en Gaule et en Germanie : les dattes}

Les vins ne sont pas les seules denrées importées de Méditerranée orientale en amphore, puisque la documentation archéologique et les sources littéraires montrent que des circuits commerciaux approvisionnaient également la Méditerranée occidentale en fruits, dont des prunes, des figues ou des dattes, retenues comme exemple ici.

L'impossibilité de faire fructifier les palmiers dattiers en Italie, et à plus forte raison en Gaule, donne à leurs fruits un indubitable caractère exotique aux yeux des consommateurs antiques (Varron, De agricultura, 2, 1 ; Pline, HN, XIII, 27 ; voir également André, 1981, p. 84). Elles sont produites en Espagne, mais les dattes n'y sont pas sucrées ; de même, Pline dit qu'en Afrique elles sont douces, mais perdent rapidement leur saveur (Pline, $H N$, XIII, 27). C'est bien en Orient, notamment au Levant (Judée, Syrie), en Arabie, en Assyrie, en Égypte ou encore à Chypre que les différentes sortes de dattes que l'on rencontre dans l'Antiquité sont les plus fameuses (Pline, HN, XIII, 41-50).

Des recherches menées sur les conteneurs levantins importés en Gaule à l'époque impériale ont montré des liens entre les dattes et certains types de récipient, comme les amphores Kingsholm 117 et Célestins 1A (Bonnet, 1998 ; Lemaître et al., 2005, p. 524-525). L'origine levantine d'une partie au moins de ces conteneurs a pu être confirmée grâce aux analyses chimiques (Lemaître et al., 2005).

Les études archéologiques publiées ces trente dernières années permettent aujourd'hui de dresser une carte des découvertes de dattes dans les sites terrestres et sous-marins de l'ouest de l'Europe [ill. 5]. Elles apparaissent en petites quantités, des Pyrénées au sud de la Grande-Bretagne et $\mathrm{du}$ Centre-Ouest aux territoires des Germanies (Carreras Monfort, Williams, 2002).

Les dattes sont déposées de manière privilégiée dans les tombes et/ou en tant qu'offrandes en contexte cultuel (Marinval, 1993). Mais un petit nombre de ces fruits est également attesté dans de rares contextes domestiques, comme à Avenches, dans la cave incendiée d'une maison entre la fin $\mathrm{du} \mathrm{II}^{\mathrm{e}}$ et le début du $\mathrm{III}^{\mathrm{e}}$ siècle de notre ère, où ils ont été retrouvés dans une amphore (Keller, 1875). La publication de Ferdinand Keller présente une planche des deux récipients, encore complets, dont l'un se distingue par sa panse côtelée associée à un fond plat annulaire et au terme explicite Datteln' [ill. 6].

La fouille de deux épaves localisées à proximité de la côte provençale, La Tradelière et Dramont D, illustre également la commercialisation des mêmes fruits exotiques, probablement en direction de 

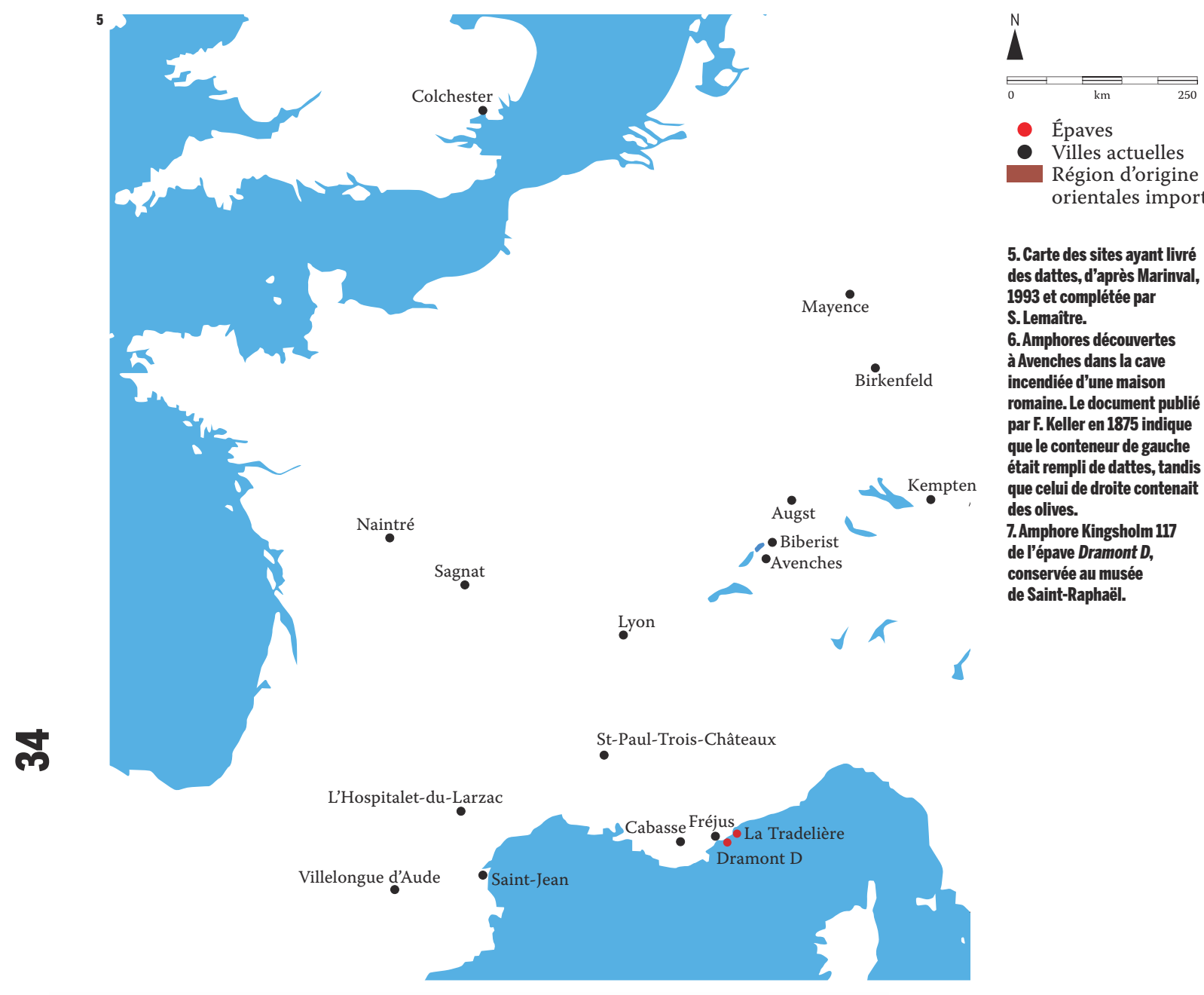

- Épaves

- Villes actuelles

Région d'origine des amphores orientales importées en Gaule

5. Carte des sites ayant livré des dattes, d'après Marinval,

1993 et complétée par

S. Lemaître.

6. Amphores découvertes

à Avenches dans la cave

incendiée d'une maison

romaine. Le document publié

par F. Keller en 1875 indique

que le conteneur de gauche

était rempli de dattes, tandis

que celui de droite contenait

des olives.

7. Amphore Kingsholm 117

de l'épave Dramont $D$,

conservée au musée

de Saint-Raphaël. 
la Gaule. Dans l'épave de La Tradelière, localisée au sud des îles de Lérins, une cinquantaine de dattes est signalée par Alex Pollino, sans que l'auteur décide si elles constituent un complément de cargaison ou une provision de bord (Pollino, 1986, p. 188). Il faut noter que l'épave a par ailleurs livré plusieurs exemplaires d'amphores Kingsholm 117. Le deuxième site sous-marin daté du milieu du ${ }^{\mathrm{er}}$ siècle, dont l'étude a livré des dattes, est l'épave Dramont $D$, située au sud-ouest de l'île d'Or près de Saint-Raphaël (Joncheray, 1972, p. 33). Les fruits ont été découverts de manière inhabituelle dans des amphores de type Dressel 2/4. Il est difficile de savoir si l'ensemble des amphores de cette forme retrouvées dans l'épave transportaient toutes des dattes (Joncheray, 1973, p. 24). La cargaison comprenait également des amphores rhodiennes contenant des graines de figues (Joncheray, 1973, pl. VI) et des récipients vides qu'il faut identifier comme des amphores levantines Kingsholm 117 [ill. 7] (Joncheray, 1973, p. 36-37). Même si la présence des restes de fruits dans des conteneurs inhabituels bouleverse quelque peu l'ordre établi, il n'empêche que l'épave du Dramont $D$ illustre bien l'arrivée par voie maritime en Gaule de fruits d'origine lointaine.
Des importations régulières de vins et de fruits à caractère exotique ont existé en Gaule dès la fin de l'époque républicaine et jusqu'au ${ }_{\text {II }}{ }^{\mathrm{e}}$ siècle : passum, dattes, figues... Ces produits sont largement mentionnés dans les sources textuelles antiques. Les indices archéologiques montrent que la commercialisation de ces denrées vers l'Occident est liée à des types d'amphore particuliers et s'est faite en quantités modestes, mais de manière régulière. Les vins exotiques ou d'outre-mer sont majoritairement importés du Sud-Ouest anatolien, tandis que l'origine levantine de certains conteneurs à fruits est maintenant confirmée par des analyses chimiques. La consommation de ces produits était sans doute réservée aux catégories aisées de la population, ce que disent les sources littéraires pour l'Italie et les contextes des découvertes archéologiques pour la Gaule romaine. Le caractère exotique de ces denrées les place souvent en haut (ou à part) de l'échelle des catégories d'aliments alors qu'ils ne sont pas obligatoirement des produits de luxe au départ. Enfin, cette enquête montre que l'introduction de denrées exotiques dans l'alimentation des habitants d'Europe occidentale est issue d'une longue tradition qui existait déjà dans l'Antiquité et qu'elle n'est donc pas apparue aux XII ${ }^{\mathrm{e}}$-XIII ${ }^{\mathrm{e}}$ siècles (Ketcham Wheaton, 1984 cité dans Régnier, 2006)

\section{Références bibliographiques}

CATON, 1975 : De l'agriculture, GoujARd R. (TRAD.), Paris, Les Belles Lettres (CUF).

DiosCoride, 1958 : De Materia medica, III, WELLMANN M. (TRAD.), Berolini.

Pline L'ANCIEN, 1956 : Histoire naturelle, XIII, ERnOUT A. (TRAD.), Paris, Les Belles Lettres (CUF).

Pline L'ANCIEN, 1958 : Histoire naturelle, XIV, ANDRÉ A. (TRAD.), Paris, Les Belles Lettres (CUF).

ANDRÉ J., 1981 : L'alimentation et la cuisine à Rome, Paris, Les Belles Lettres.

Becker C., Constantin C., Desbat A., Jacquin L., LASCOUX J.-P., 1986 : « Le dépôt d'amphores augustéen de la rue de la Favorite à Lyon ", Figlina, 7 , p. 65-89.

BonNet C., 1998 : « Nouvelles formes d'amphores orientales, place des Célestins à Lyon ? », in LAUbenheimer F. (DIR.), Les amphores en Gaule - II Production et circulation, Besançon, Presses universitaires franc-comtoises (coll. Série Amphores), p. 237-244.

Carreras Monfort C., Williams D.F., 2002 : «"Carrot" Amphoras : a Syrian or Palestinian Connection?" in HuMPHREY J. H. (ÉD), The Roman and Byzantine near East, vol. 3, p. 133-144 (suppl. au Journal of Roman Archeology, $\mathrm{n}^{\circ}$ 49).
Delor A., Roms C., 2007: « Datations céramiques et datations absolues : le cas de la place de la Libération à Troyes (Aube) », in SFECAG, Actes du Congrès de Langres, p. 71-96.

IMBERT E. L., 2002 : « Du goût de nos ancêtres pour le bon vin : des amphores de Cnide à Telo Martius ", Revue du Centre archéologique du Var, p. 72-96.

JONCHERAY J.-P., 1972 : « Contribution à l'étude de l'épave Dramont $\mathrm{D}$, dite "des pelvis" d'après les travaux du groupe d'étude sous-marines de Saint-Raphaël », Cahiers d'archéologie subaquatique, 1, p. 11-34.

JoNCHERAY J.-P., 1973 : « Contribution à l'étude de l'épave Dramont D (campagnes 1970-1971) », Cahiers d'archéologie subaquatique, 2, p. 9-47.

Keller F., 1875: "Südfrüchte aus Aventicum », Anzeiger für schweizerische Geschichte und Altertumskunde, 1 , p. 580-581.

Ketcham Wheaton B., 1984 : L'office et la bouche, Paris, Calmann-Lévy.

LeblanC O., Desbat A., 1992 : « Un lot de céramiques du début du III ${ }^{\mathrm{e}}$ siècle à Saint-Romain-en-Gal », $R A N$, vol. 25, p. $125-150$.

LEMAÎTRE S., 2002: « Recherche sur la diffusion en Gaule, des amphores produites dans le sud-ouest de la Turquie, à l'époque impériale », in BLONDE F., BALLET P., SALLES J.-F. (DIR.), Céramiques hellénistiques et romaines. Productions et diffusion en Méditerranée orientale (Chypre, Égypte et côtesyro-palestinienne), Actes du colloque de Lyon (2-4 mars 200o), Lyon, Maison de l'Orient Méditerranéen Jean Pouilloux (coll.Travaux de la Maison del'Orient Méditérranéen, 35), p. 213-226

LEMAÎTRE S., 2005 : «Des vins grecs à Lugdunum », in Desbat A. (DIR.), Lugdunum, naissance d'une capitale, Gollion, Infolio, p. 167-168.

LEMAîtrRe S. (avec la collaboration de ARQUE M.-C. AudE V.), 2012 : « Pour qui sont ces amphores qui gisent sur vos sites. Importations méditerranéennes à Poitiers-Lemonum ", in SFECAG, Actes du Congrès de Poitiers, p. 181-195.
LEMAÎTRE S., à paraître: Saveurs et denrées orientales en Gaule romaine, d'Auguste à la fin du III' siècle ap. J.-C. Les données du mobilier amphorique, Oxford, Archaeopress (coll. Roman and Late Antique Mediterranean Pottery).

Lemaître S., Waksman Y., Roumie M., Nsouli B., REYNOLDS P., 2005: «À propos de l'origine levantine de plusieurs types d'amphores importés en Gaule à l'époque impériale », in SFECAG, Actes du Congrès de Blois, p. 515-528.

MARANGOU-LerAt A., 1995 : Le vin et les amphores de Crète de l'époque classique à l'époque impériale, Athènes, École française d'Athènes (coll. Études crétoises, 30).

MARINVAL P., 1993: «Étude carpologique d'offrandes alimentaires végétales dans les sépultures galloromaines : réflexions préliminaires », in FERDIERE A. (DIR.), Monde des morts, monde des vivants en Gaule rurale, Actes du colloque ARCHEA/AGER, Orléans, 7-9 février 1992, Tours, FERACP (coll. Suppl. à la Revue archéologique du Centre, 6), p. 45-65.

MARTIN-KILCHER S., 1994: Die römischen Amphoren aus Augst und Kaiseraugst (Forschungen in Augst 7/2-3).

Pollino A., 1986: «L'épave de La Tradelière », in L'exploitation de la mer. La mer, moyen d'échange et de communication, $V^{e}$ Rencontres internationales d'archéologie et d'histoire, Antibes, octobre 1985 , Juan-les-Pins, APDCA, p. 171-189.

RÉGNIER F., 2004: L'exotisme culinaire. Essai sur les saveurs de l'Autre, Paris, Presses universitaires de France.

RÉGNIER F., 2006 : « Le monde au bout des fourchettes : voyage dans l'exotisme culinaire », www.lemangeurocha.com, juin 2006.

TCHERNIA A., 1986 : Le vin de l'Italie romaine. Essai d'histoire économique d'après les amphores, Rome, Publications de l'École française de Rome (coll. BEFAR, 261).

TCHernia A., 2011: Les Romains et le commerce, Naples, Centre Jean Bérard (coll. Études, 8). 